\title{
Microwave Irradiation Effect on Structural, Optical, and Thermal Properties of Cadmium Oxide Nanostructure
}

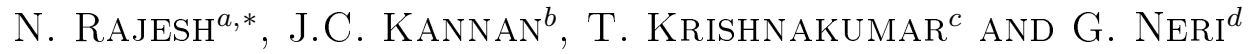 \\ ${ }^{a}$ Department of Physics, KSR College of Engineering, Tiruchengode, Tamilnadu - 637215, India \\ ${ }^{b}$ Department of Physics, KSR Institute for Engineering and Technology, Tiruchengode, Tamilnadu - 637215, India \\ ${ }^{c}$ Department of Physics, Tagore Institute of Engineering and Technology, Attur, Salem, Tamilnadu - 636112, India \\ ${ }^{d}$ Department of Industrial Chemistry and Materials Engineering, University of Messina, 98166 Messina, Italy
}

(Received September 14, 2013)

Cadmium oxide nanostructures were prepared by microwave assisted wet chemical technique at different time intervals. Results on structural, optical, and thermal properties of the CdO nanostructures as a function of the microwave irradiation were reported. The X-ray diffraction data indicates that the sample showed perfection in the microstructural improvement as a function of microwave irradiation. Surface morphological changes with different time of microwave irradiation were recorded by transmission electron microscope and the particle size were found in the ranges from 5 to $30 \mathrm{~nm}$. Chemical composition and thermal stability of the samples were analyzed by energy dispersive X-ray spectrum and thermogravimetric analysis, respectively. The band gaps were shifted towards the blue region due to the Moss-Burstein effect and it exhibited direct band transitions, which corresponds to optical band gaps of 3.92-4.20 eV and contrast behavior of optical properties of CdO nanostructure in UV and IR regions were registered. Room temperature photoluminescence spectra revealed that the intensity of luminescent emission tends to decrease with the increase in exposure of microwave irradiation.

DOI: 10.12693/APhysPolA.125.1229

PACS: 81.07.-b, 68.37.Lp, 78.67.-n, 81.70.Jb

\section{Introduction}

In the past decade, nanocrystallites have attracted more attention in both fundamental research and practical application for their quantum confinement phenomenon. It is generally known that nanostructured materials display unusual physical properties that differ from those of bulk materials and are promising for the fabrication of novel devices. In recent years, a wide range of nanosized powders have been synthesized to engineer desired properties such as chemical, electrical, mechanical, and optical properties [1].

Nowadays semiconductor nanostructures attract a lot of interest due to their wide applications in the electrical and optoelectronic devices. From the optical point of view the interest is connected with a possibility of tuning the light emission from semiconductor nanocrystals (NCs) by varying their size, owing to the effect of quantum confinement [2]. The use of transparent conducting oxides (TCO) in optoelectronic and photovoltaic devices has stimulated research on this field in recent years.

In particular, cadmium oxide is a promising material for solar cell application [3-5] and photodiodes [6]. A variety of techniques have been reported to make $\mathrm{CdO}$ nanostructures like sol-gel [7], DC magnetron sputtering [8], radio-frequency sputtering [9], spray pyrolysis [10], pulsed laser deposition [11], chemical vapor de-

*corresponding author; e-mail: rajeshkarur83@gmail.com position [12], chemical bath deposition [13], hydrothermal [14] and solvothermal [15] but few works on the preparation and characterization of $\mathrm{CdO}$ nanoparticles by microwave assisted technique. In this contrast, our focus arises toward synthesis of cadmium oxide using microwave assisted wet chemical route, because microwaves are directly coupled with the material result in rapid and uniform heating. In the present work, we have registered the changes of structural and optical properties (reflectance, band gap, absorption and extinction coefficient, refractive index, dielectric constant and optical conductivity) of cadmium oxide nanostructure with the effect of microwave irradiation at three different time intervals. Several researchers have reported that $[8,16-18]$ the optical properties of $\mathrm{CdO}$ nanoparticles have been achieved by the process of annealing with huge time (i.e., require high energy consumption) but in our present method we achieved good quality crystalline $\mathrm{CdO}$ nanostructures with fine optical property within 5-15 min with low energy consumption. Up to our knowledge, there is not any reported data about the vast optical constants of cadmium oxide samples with effect of microwave irradiation based on the diffused reflectance calculations.

\section{Experimental}

\subsection{Synthesis}

In order to prepare $\mathrm{CdO}$, precursor solution cadmium acetate dihydrate $\left(\mathrm{Cd}\left(\mathrm{COOCH}_{3}\right)_{2} \cdot 2 \mathrm{H}_{2} \mathrm{O}\right)$ with a concentration of $0.1 \mathrm{M}$ was prepared separately. Subsequently, ammonia $\left(\mathrm{NH}_{4} \mathrm{OH}\right)$ solution was added to the above solution. The resulting mixture was stirred at room tem- 
perature until the $\mathrm{pH}$ of the solution is 8 . The resulting precipitate was washed with double distilled water. The obtained precipitate was placed in a microwave oven $(2.45 \mathrm{GHz}, 800 \mathrm{~W})$ and irradiated for 5 min (sample A), $10 \mathrm{~min}$ (sample B) and $15 \mathrm{~min}$ (sample C), and finally the precipitate was filtered and dried at $120^{\circ} \mathrm{C}$.

\subsection{Characterization}

The crystalline structure of the samples was analyzed by X-ray diffraction (XRD) using a Bruker AXSD8 Advance instrument and using the $\mathrm{Cu} K_{\alpha}$ wavelength of $1.5406 \AA$. The average crystallite size of the $\mathrm{CdO}$ were evaluated using the Scherrer formula $d=k \lambda / \beta \cos \theta$, where $d$ is the mean crystalline size, $k$ is a grain shape dependent constant (0.9), $\lambda$ is the wavelength of the incident beam, $\theta$ is a Bragg reflection angle, and $\beta$ is the full width half maximum. Transmission electron microscopy (TEM) analysis was performed on a Philips instrument Model CM12 operating at $120 \mathrm{kV}$ and directly interfaced with a computer for real-time image processing. Energy dispersive X-ray spectroscopy (EDX) analysis was observed by JEOL5600LV microscope at an accelerating voltage of $10 \mathrm{kV}$. The difused reflectance spectroscopy (DRS) reflectance spectra were studied using UV-DRS absorption spectroscopy - Specord S600-212C205 UV Spectrophotometer. Photoluminescence spectrum was carried out using Spectroflurometer - Fluorolog - FL3-11. Thermal stability of the sample was examined by using a Perkin Elmer Diamond TGA instrument under $\mathrm{N}_{2}$ atmosphere.

\section{Results and discussion}

\subsection{Structural characterizations}

Figure $1 \mathrm{a}-\mathrm{c}$ shows the X-ray diffraction pattern of microwave irradiated $\mathrm{CdO}$ nanostructure with different time intervals i.e., 5 (sample A), 10 (sample B), and 15 (sample C) min, respectively. From the figure, formation of crystalline $\mathrm{CdO}$ phase has been clearly registered. In fact, the sharp peaks indicate that the irradiated samples $\mathrm{A}, \mathrm{B}$ and $\mathrm{C}$ possess high crystallinity. Further, no traces of impurity phases such as $\mathrm{CdO}_{2}, \mathrm{Cd}(\mathrm{OH})_{2}$, and $\mathrm{CdCO}_{3}$ are detected in the XRD pattern, indicating the formation of $\mathrm{CdO}$ crystalline phase. The as-prepared samples exhibited the following Miller indices [111], [200], [220], [311], and [222] which is matched well with ICDD card \#65-2908 and face-centered cubic structure of $\mathrm{CdO}$ [17]. The average crystalline size of the cadmium oxide crystals were calculated from the Scherrer equation and it was found to be 25 to $51 \mathrm{~nm}$ i.e., the average crystalline size increases when exposing the microwave irradiation time increases.

In general, microwave synthesis is quite fast, simple, and very energy efficient. The exact nature of microwave interaction with reactants during the synthesis of materials is somewhat unclear and speculative. However, transfer of energy from microwaves to the material is believed to occur either through resonance or relaxation, which

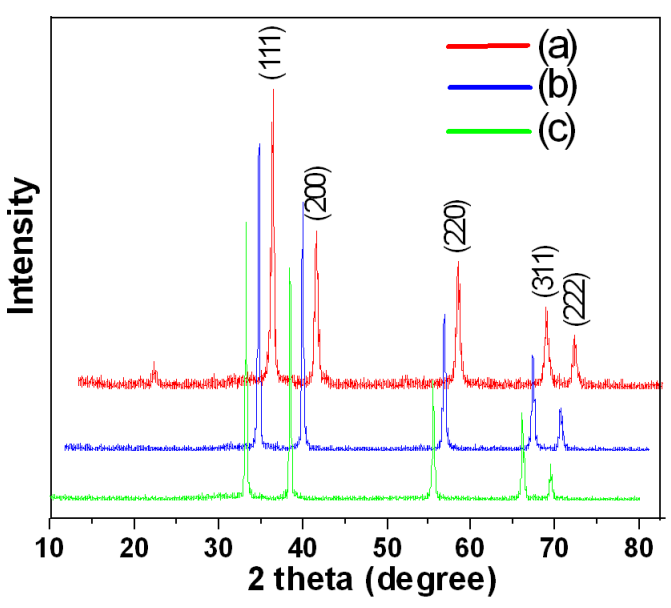

Fig. 1. XRD pattern of the $\mathrm{CdO}$ nanostructure samples A, B, and C.

results in rapid heating and also greatly accelerated the nucleation of the crystalline $\mathrm{CdO}$ formed. The amount of defects can be estimated by calculating the dislocation line density $(\delta)$ which is related to particle size $(D)$ as

$$
\delta=n / D^{2} \text {. }
$$

From this relation [19-21], it is obvious that as the particle size increases, the dislocation density decreases. In our case, microwave irradiation increases, the dislocation line density decreases depicting the reduction of defect density, which implies improved crystallinity.

\subsection{Surface morphological analysis}

The surface morphology of $\mathrm{CdO}$ nanostructures of samples $\mathrm{A}, \mathrm{B}$, and $\mathrm{C}$ were recorded by TEM microscope as shown in Fig. 2a,c,e. The microwave irradiation with the function of time on cadmium hydroxide solution accelerates the surface structure. Agglomerated irregular shaped $\mathrm{CdO}$ nanostructures were obtained when exposure of microwave irradiation time was 5 min (Fig. 2a) and an irradiation time was increased $10 \mathrm{~min}$ then irregular shapes were transformed into spherical shape (Fig. 2c). Finally rod like shape structures are obtained when microwave irradiation increased for $15 \mathrm{~min}$ in the range of 5-30 nm. The thickness and length of nano rod has $25-40 \mathrm{~nm}$ and of few $\mu \mathrm{m}$ (Fig. 2e). From the observed behavior one can suggest increase of the exposing time of microwave irradiation on cadmium hydroxide solution tunes the surface morphology of CdO. The EDX spectrum of $\mathrm{CdO}$ nanostructures of samples $\mathrm{A}, \mathrm{B}$, and $\mathrm{C}$ were shown in Fig. 2b,d,f. The compositional analysis of the samples (A, B, and C) was confirmed by EDX analysis and the results are shown in Fig. 2b,d,f. The $\mathrm{Cd}$ and $\mathrm{O}$ peaks are present in the EDX spectra and this confirms the formation of $\mathrm{CdO}$.

\subsection{Optical studies (DRS)}

The optical constants of the microwave irradiated cadmium oxide nanostructure samples $\mathrm{A}, \mathrm{B}$, and $\mathrm{C}$ were determined by UV-DRS spectra (before that, samples were 

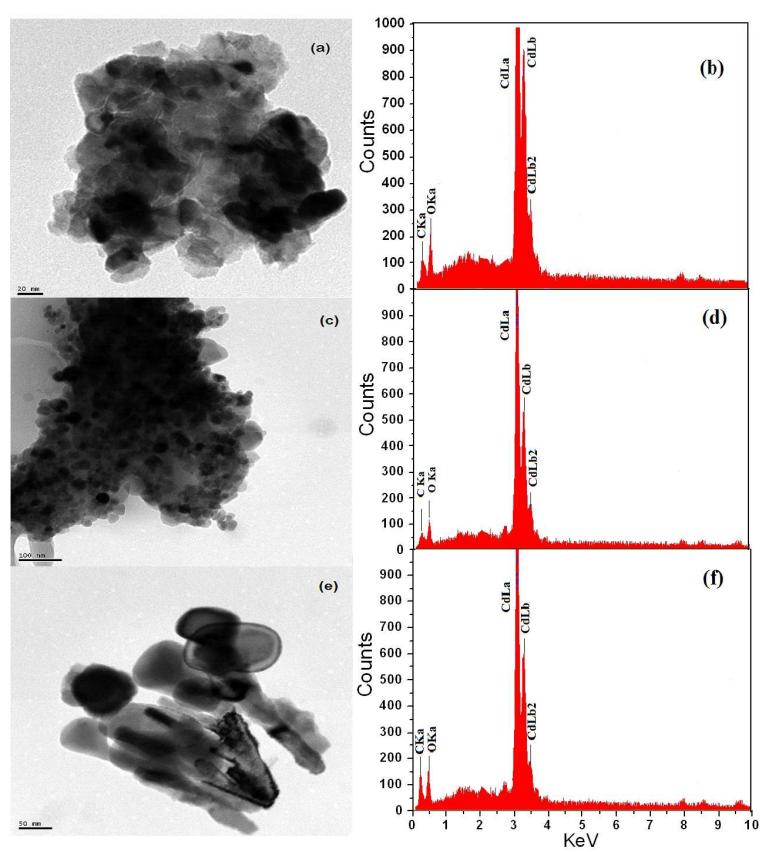

Fig. 2. TEM images (a, c,e) and corresponding EDX pattern $(b, d, f)$ of the samples A, B, and C.

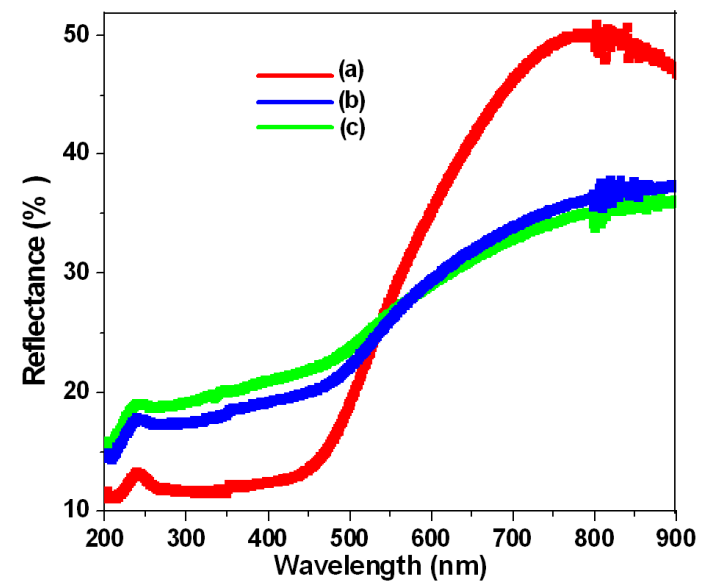

Fig. 3. Diffused reflectance spectrum as a function of wavelength for $\mathrm{CdO}$ samples $\mathrm{A}, \mathrm{B}$, and $\mathrm{C}$.

prepared by thick film on glass substrate). From Fig. 3 it is clear that the reflectance of the samples increases with increasing wavelength. Minimum reflectance is observed in UV region then it starts to increase in visible region and reaches maximum in IR region. Exposure of microwave irradiation time increases causes reflectance increases in UV region but it exhibits opposite behavior in IR region i.e., as the microwave irradiation time increases the reflectance value decreases.

To obtain the optical band gap of the microwave irradiated $\mathrm{CdO}$ samples at room temperature, the KubelkaMunk theory is generally used and it is expressed by the following relation [22]:

$$
F(R)=\frac{(1-R)^{2}}{2 R},
$$

where $F(R)$ is the Kubelka-Munk function and $R$ is the reflectance. The electronic transition between the valence and conduction bands starts at the absorption edge corresponding to the minimum energy difference between the lowest energy of the conduction band and the highest energy of the valence band in crystalline materials. The optical band gap was determined from the relation, $(\alpha h \nu)=\left(h \nu-E_{\mathrm{g}}\right)^{n}$, where $\alpha$ is the linear absorption coefficient of the material, $E_{\mathrm{g}}$ is the optical band gap and $n$ is a constant which determines the type of optical transitions: for $n=1 / 2,3 / 2,2$, and 3 depending on the nature of the electronic transition responsible for the absorption: $n=1 / 2$ for allowed direct transition, $n=2$ for allowed indirect transition, $n=3 / 2$ for forbidden direct transition and $n=3$ for forbidden indirect transition [23-25]. $F(R)$ is directly proportional to the absorbance therefore; $F(R)$ values were converted to the linear absorption coefficient by means of the $\alpha=$ absorbance $/ t=F(R) / t$ relation, where $t$ is the thickness of the film. The curve of $[F(R) h \nu / t]^{1 / 2}$ vs. $h \nu$ for the $\mathrm{CdO}$ samples A, B, and C was plotted [26] as shown in Fig. 4. The direct band gap values were determined by extrapolating the linear portions of these graphs to the energy axis at $F(R)=0$ and was found to be $3.92,4.09,4.20 \mathrm{eV}$ for samples A, B, and $\mathrm{C}$ respectively, i.e., the microwave irradiation time increase causes the band gap value increase. The obtained values are higher than the bulk $\mathrm{CdO}$ [27-30]. The higher direct band gap value with effect of microwave irradiation in the present work is believed to be partly due to the Moss-Burstein (M-B) effect. The blue-shift of the absorption edge has been generally interpreted by the M-B effect $[22,31]$. The higher value of optical band gap arises due to improvement in the crystallinity of $\mathrm{CdO}$ during microwave irradiation and this confirms the quantum confinement. Several researchers $[7,16,17,30]$ obtained band gap values of $2.29 \mathrm{eV}$ at $450{ }^{\circ} \mathrm{C}$ for $120 \mathrm{~min}$, $2.26 \mathrm{eV}$ at $400{ }^{\circ} \mathrm{C}, 2.7 \mathrm{eV}$ at $623 \mathrm{~K}$ for $2 \mathrm{~h}, 2.79-2.50 \mathrm{eV}$ at $200^{\circ} \mathrm{C}-450^{\circ} \mathrm{C}$ for $\mathrm{CdO}$ samples by various methods. But the present work yields the higher band gap value of $\mathrm{CdO}$ nanostructure than bulk $\mathrm{CdO}$ without post synthesis of annealing and low energy consumption suggests the applicability of the material under this method in optoelectronic field.

The refractive index $(n)$ and extinction coefficient $(k)$ of the $\mathrm{CdO}$ samples $(\mathrm{A}, \mathrm{B}, \mathrm{C})$ can be calculated by using following relation $[32,33]$ :

$$
\begin{aligned}
& n=\left(\frac{1+R}{1-R}\right)+\left[\frac{4 R}{(1-R)^{2}}-k^{2}\right]^{1 / 2}, \\
& k=\frac{\alpha \lambda}{4 \pi} .
\end{aligned}
$$

Figure 5 shows the dependence of the refractive index of the microwave irradiated $\mathrm{CdO}$ samples on wavelength. The refractive index of all samples lies between 2 and 5.99. The dispersion curve of refractive index is lower in the UV region and rises rapidly towards IR re- 


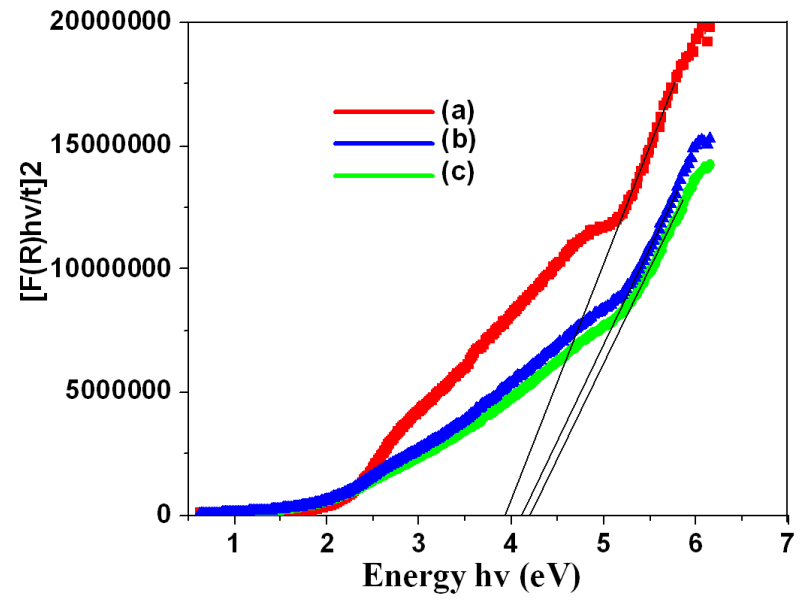

Fig. 4. Plotting of (a) $\left[F\left(R_{\infty}\right) h \nu\right]^{2}$ as a function of the photon energy for CdO samples A, B, and C.

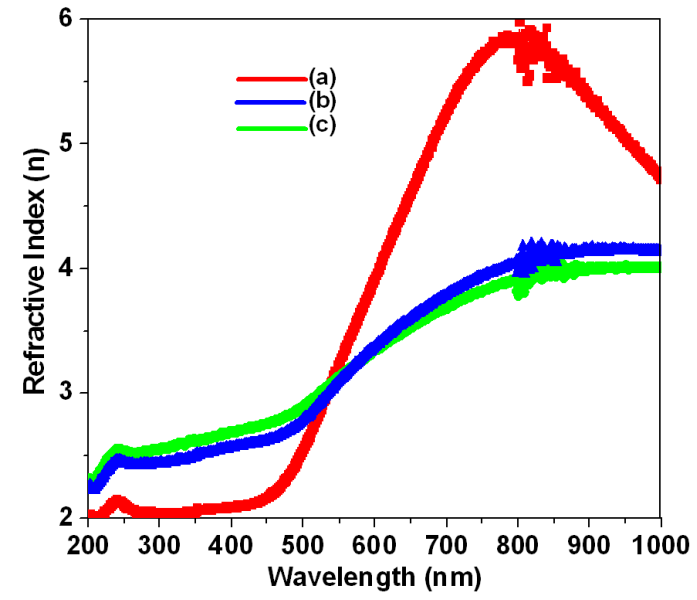

Fig. 5. Variation of the refractive index as a function of wavelengths for $\mathrm{CdO}$ samples $\mathrm{A}, \mathrm{B}$, and C.

gion. Refractive index increases with microwave irradiation time increases in UV region and refractive index decreases with microwave irradiation time increases in IR region; this is due to the major contribution of electronic transitions for various time intervals of microwave irradiation and this may lead to a significant change in the optical parameters. The gradual increase of refractive index with wavelength implies that the normal dispersion. The spectral behavior of refractive index is quite similar to that of reflection spectrum as can be expected since the extinction coefficient values are very small.

Figure $6 \mathrm{a}$ shows the variation of absorbance coefficient $(\alpha)$ with wavelength $(\lambda)$ for microwave irradiated $\mathrm{CdO}$ samples. The calculated values of absorption coefficient are in the order of $10^{2} \mathrm{~cm}^{-1}$. From Fig. $6 \mathrm{a}$, it is seen that the absorbance coefficient increases with the decrease in wavelength. In UV region absorbance coefficient gets increased from minimum exposure of microwave irradiation to maximum exposure of microwave irradiations; in contradictory to this in IR region absorbance coefficient is maximum for minimum microwave irradiation exposure time and decreases gradually as the exposure time increases and sample A exhibits more transparence in IR region than sample $\mathrm{B}$ and $\mathrm{C}$. Figure $6 \mathrm{~b}$ shows the variation of extinction coefficient $(k)$ with wavelength $(\lambda)$ for microwave irradiated $\mathrm{CdO}$ samples. It is clear from Fig. $6 \mathrm{~b}$ that the values of $k$ changed in the range 0.00010 0.00025 and that the values of $k$ increase with increase in wavelength. The low value of extinction coefficient represents samples possessing high transmittance.

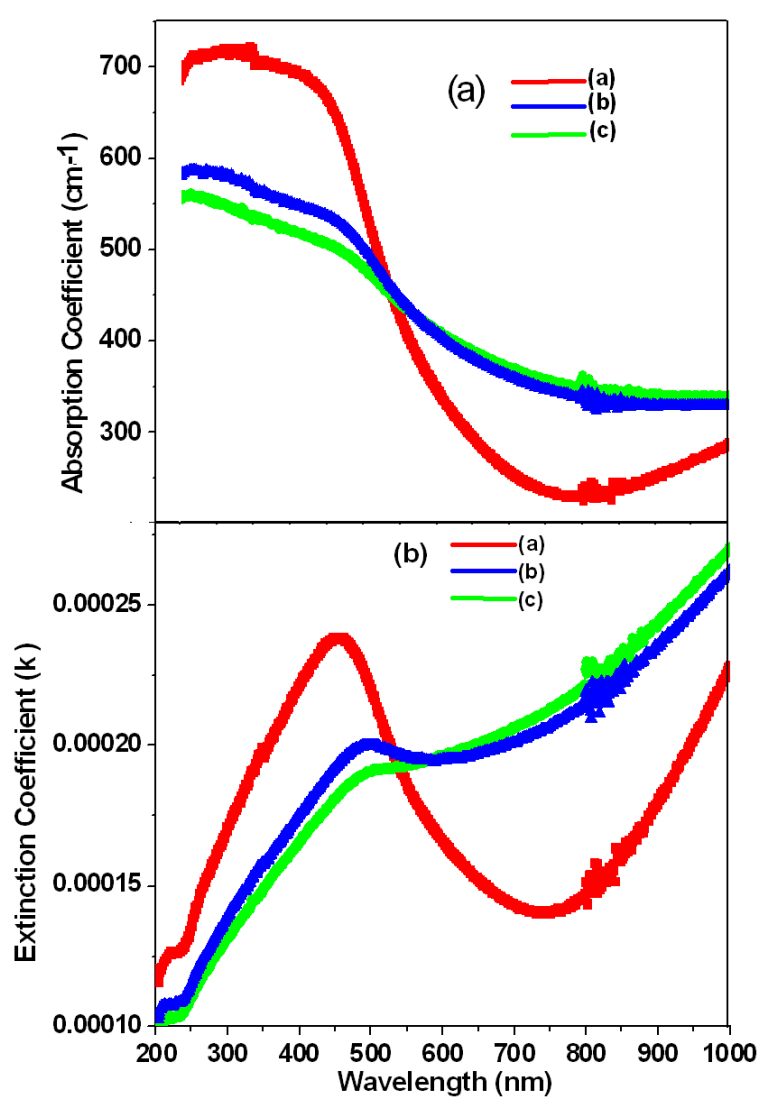

Fig. 6. Variation of absorption coefficient (a) and extinction coefficient (b) with wavelength of the CdO samples A, B, and C.

The frequency dependence of the complex electronic dielectric constant is defined as $\varepsilon(\omega)=\varepsilon_{1}(\omega)+\mathrm{i} \varepsilon_{2}(\omega)$. These two contributions represent the in-phase $\left(\varepsilon_{1}\right)$ and out-of-phase $\left(\varepsilon_{2}\right)$ components of the frequency response of the medium. The in-phase component results in dispersion, physically this means refraction of the electromagnetic radiation as it passes through the medium and the out-of-phase component gives rise to absorption. Real and imaginary parts of the dielectric constant are related to the $n$ and $k$ values. The $\varepsilon_{1}$ and $\varepsilon_{2}$ values were calculated using the formulae $[34,35]$ :

$$
\begin{aligned}
& \varepsilon_{1}=n^{2}-k^{2}, \\
& \varepsilon_{2}=2 n k .
\end{aligned}
$$


The variation of $\varepsilon_{1}$ and $\varepsilon_{2}$ values of the microwave irradiated $\mathrm{CdO}$ samples $\mathrm{A}, \mathrm{B}$, and $\mathrm{C}$ with wavelength is shown in Fig. 7a,b. It can be seen in Fig. 7a that the real part dielectric constant is less in UV region, then it starts increasing with increase in wavelength. The variations of dielectric constant with wavelength may be due to the presence of all types of polarizations namely, space charge, orientational, electronic and ionic polarization, and its losses. The characteristic of low dielectric constant suggests that the sample possesses optical quality with lesser defects and this parameter is highly important for making optoelectronic materials. It is seen from Fig. 7a that the spectral variation of $\varepsilon_{1}$ is quite similar to those of the refractive index. This is an expected situation, since extinction coefficient is too small $(\approx 0.0020$ in maximum) to make any significant contribution to the variation of real part of dielectric coefficient. An imaginary part of the dielectric constant is increased with wavelength increases as shown in Fig. $7 \mathrm{~b}$.

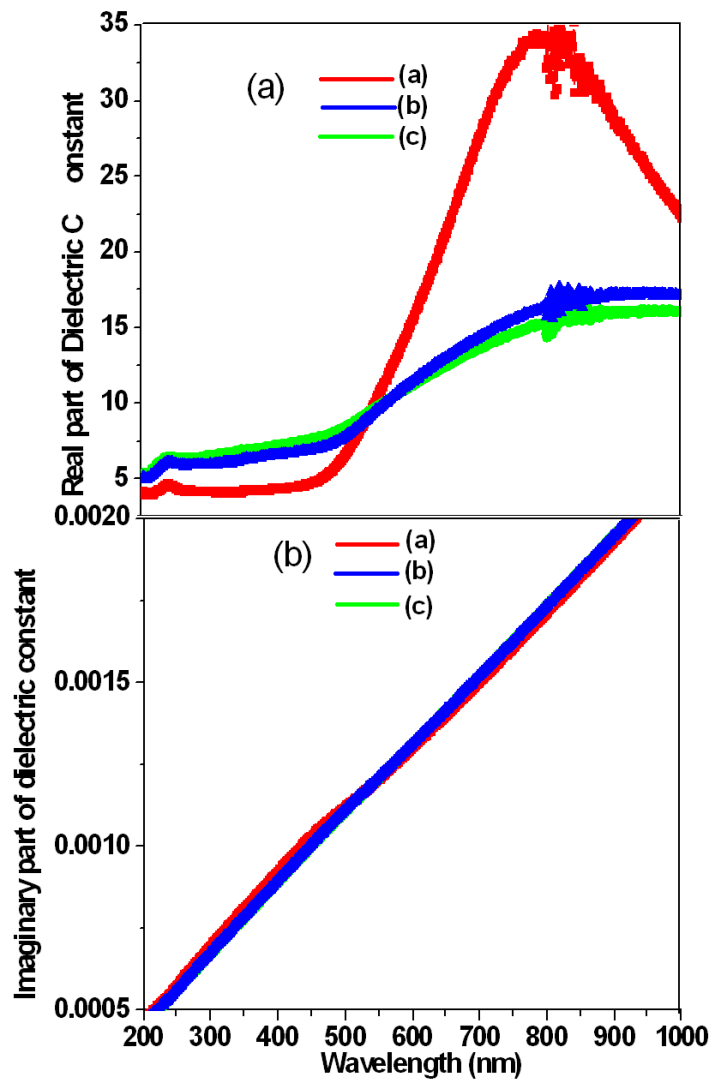

Fig. 7. The variation of real part (a) and imaginary part (b) of the dielectric constant with wavelength of the samples A, B, and C.

The real $\left(\sigma_{1}\right)$ and imaginary $\left(\sigma_{2}\right)$ parts of the optical conductivity are expressed by [36]:

$$
\begin{aligned}
& \sigma_{1}=\omega \varepsilon_{2} \varepsilon_{0}, \\
& \sigma_{2}=\omega \varepsilon_{1} \varepsilon_{0},
\end{aligned}
$$

dielectric constant. The dependence of the real and imaginary parts of the optical conductivity on the wavelength is shown in Fig. 8a,b. Figure 8a shows that the maximum real part of the optical conductivity is observed in UV region then decreases towards IR region. Figure $8 \mathrm{~b}$ shows that the minimum and maximum imaginary part of the optical conductivity is observed in UV and IR regions, respectively, then it starts to decrease in the higher wavelength. The variations of optical conductivity due to reduction of polarization and crystal defects are created by thermal activation with exposure of microwave irradiation at different time intervals. The variation of the optical conductivity may be the availability of impurities and vacancies in the crystal lattice due to exposure of microwave irradiation at different time intervals.

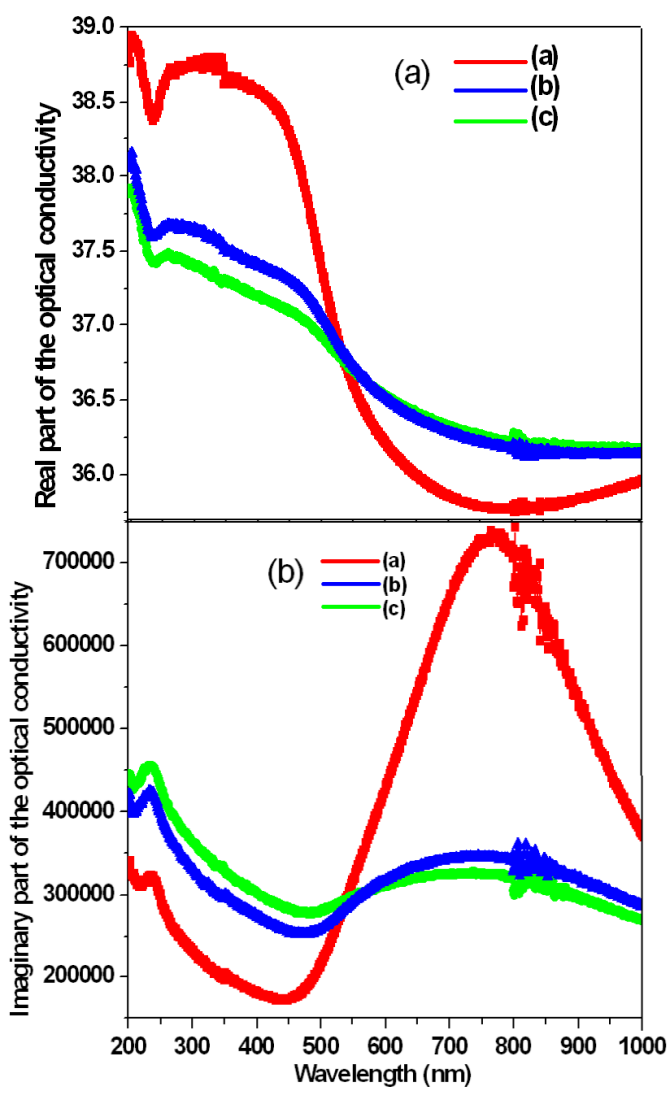

Fig. 8. The variation of real part (a) and imaginary part (b) of the optical conductivity with wavelength of the samples A, B, and C.

As can be seen from Figs. 3-8 it is demonstrated that the optical properties of sample A varying from sample $\mathrm{B}$ and $\mathrm{C}$. One can also evident from Figs. 3-8 that the optical properties of $\mathrm{CdO}$ nanostructures in $\mathrm{UV}$ region are opposite to that of IR region and the significant difference occurrence may be exposure of microwave irradiation at different time intervals. According to Eq. (1), crystal defect decreases due to increase of particle size when the microwave irradiation time increases. When the material was irradiated by microwave the vacancies, 
foreign impurities incorporated into the lattice and the molecular structure will change and this change is combined with the polarization properties of the material which will have an influence on the thermal coefficient of dielectric constant of the material. Usually, dielectric materials have permanent dipoles. As microwave irradiation increases, the molecules in the dielectric have more thermal energy and therefore, the amplitude of random motion is greater. This means that the molecules are less closely aligned with each other (even in the presence of an electric field). Hence, the optical property gets altered.

\subsection{Photoluminescence studies}

The photoluminescence (PL) spectra of microwave irradiated cadmium oxide samples $\mathrm{A}, \mathrm{B}$, and $\mathrm{C}$ are shown in Fig. 9. From the figure, it is clearly evident that all the samples exhibited the strong emission peak at $529 \mathrm{~nm}$ $(2.3 \mathrm{eV})$. Zaien and co-workers [37] reported that the emission peak of bulk $\mathrm{CdO}$ is $491 \mathrm{~nm}(2.5 \mathrm{eV})$. This red-shift from 2.3 to $2.5 \mathrm{eV}$ was caused by the formation of donor levels (the Fermi levels) near the conduction band of the $\mathrm{CdO}$ nanostructures [37]. Moreover, it can be seen from Fig. 9 samples $\mathrm{B}$ and $\mathrm{C}$ show the similar spectra but it is different from sample A that the intensity of luminescent emission decreases with increase in the exposure of microwave irradiation time, and it is attributed to the decrease in oxygen vacancies as a function of microwave irradiation.

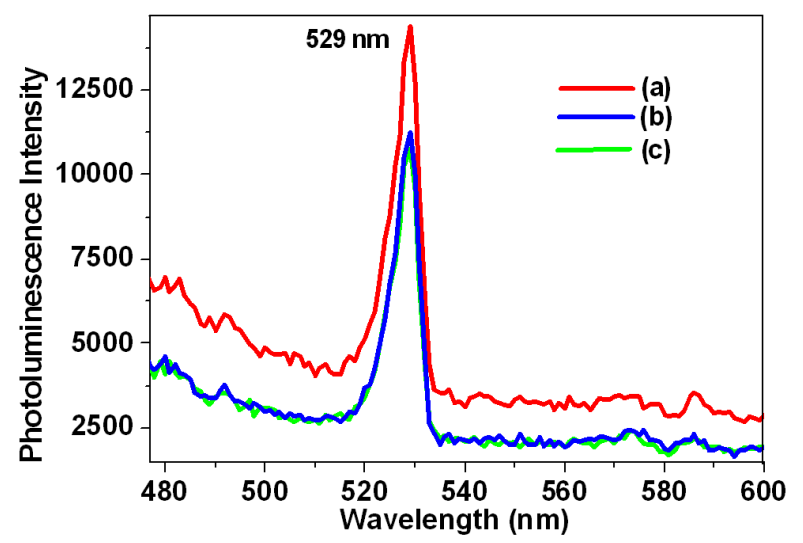

Fig. 9. Photoluminescence spectrum of $\mathrm{CdO}$ nanostructure samples A, B, and C.

\subsection{Thermogravimetric analysis}

Thermogravimetric analysis was recorded for as-prepared cadmium oxide nanostructure heated to $700^{\circ} \mathrm{C}$ with a ramp rate of $20^{\circ} \mathrm{C} / \mathrm{min}$ under $\mathrm{N}_{2}$ atmosphere and is shown in Fig. 10. The first weight loss of about $3 \%$ occurring from room temperature to $220^{\circ} \mathrm{C}$ could be attributed to the release of surface adsorbed water [38]. The second major weight loss happening at $220-350^{\circ} \mathrm{C}$ and weight loss of $12.4 \%$ was measured, corresponding to release of water molecule from interior of cadmium oxide nanostructure [39]. The third weight loss $7.7 \%$ at $600{ }^{\circ} \mathrm{C}$ may be related to the decomposition of organic groups in the precursor [38]. There is no weight loss observed after $550{ }^{\circ} \mathrm{C}$, implying the as-synthesized $\mathrm{CdO}$ is stable up to this temperature.

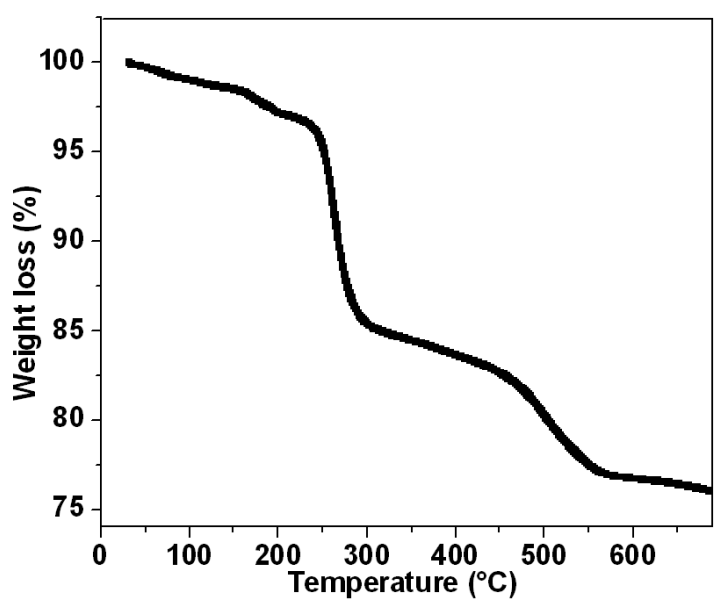

Fig. 10. TGA spectra of sample $\mathrm{A}$ in $\mathrm{N}_{2}$ atmosphere.

\section{Conclusion}

This work describes a novel approach to synthesis of cadmium oxide nanostructure by microwave assisted wet chemical technique at different time intervals. XRD data indicates that samples show microstructural perfection improvement as a function of microwave irradiation and confirms face-centered cubic structure of the samples. From the behavior observed from TEM micrograph, it is possible to tune the morphology of the $\mathrm{CdO}$ nanostructure when microwave irradiation time increases. The peaks of $\mathrm{Cd}$ and $\mathrm{O}$ show that the formation of $\mathrm{CdO}$ was confirmed by EDX. Contradicting optical properties of $\mathrm{CdO}$ in UV and IR region were recorded by DRS spectrum. The photoluminescence study showed decrease in the luminescent emission with the increase in exposure of microwave irradiation. By tuning the microwave irradiation, one can get required higher optical band gap of $\mathrm{CdO}$ for required optoelectronic applications.

\section{References}

[1] E.C.C. Souza, J.F.Q. Rey, E.N.S. Muccillo, Appl. Surf. Sci. 255, 3779 (2009).

[2] X. Peng, L. Manna, W. Yang, J. Wickham, E. Scher, A. Kadavanich, A.P. Alivisatos, Nature 404, 59 (2000).

[3] T.L. Chu, S.S. Chu, J. Electron. Mater. 19, 1003 (1990).

[4] C.H. Champness, C.H. Chan, Sol. Energy Mater. Sol. C 37, 75 (1995).

[5] A.A. Al-Qurani, C.H. Champness, in: Proc. 26th IEEE Photovoltaic Specialists Conf., Anaheim, IEEE, Picataway (NJ) 1997, p. 415. 
[6] R. Kondo, H. Okhimura, Y. Sakai, Jpn. J. Appl. Phys. 10, 1547 (1971).

[7] J.S. Cruz, G.T. Delgado, R.C. Perez, S.J. Sandoval, O.J. Sandoval, C.I.Z. Romero, J.M. Marin, O.Z. Angel, Thin Solid Films 493, 83 (2005).

[8] T.K. Subramanyam, S. Uthanna, B.S. Naidu, Mater. Lett. 35, 214 (1998).

[9] N. Ueda, H. Maeda, H. Hosono, H. Kawazoe, J. Appl. Phys. 84, 6174 (1998).

[10] B.J. Lokhande, P.S. Patil, M.D. Uplane, Mater. Chem. Phys. 84, 238 (2004).

[11] M. Yan, M. Lane, C.R. Kannewurf, R.P.H. Changa, Appl. Phys. Lett. 78, 2342 (2001).

[12] X. Liu, C. Li, S. Han, J. Han, C. Zhou, Appl. Phys. Lett. 82, 1950 (2003).

[13] L.R. Gutierrez, J.J.C. Romero, J.M.P. Tapia, E.B. Calva, J.C.M. Flores, M.O. Lopez, Mater. Lett. 60, 3866 (2006).

[14] K.C. Song, Y. Kang, Mater. Lett. 42, 83 (2000).

[15] L. Korosi, S. Papp, V. Meynen, P. Cool, E.F. Vansant, I. Dekany, Physicochem. Eng. Aspects 268, 147 (2005).

[16] O. Vigil, F. Cruz, A. Morales-Acevedo, G. ContrerasPuente, L. Vaillant, G. Santana, Mater. Chem. Phys. 68, 249 (2001).

[17] C.H. Bhosale, A.V. Kambale, K.Y. Kokate, Mater. Sci. Eng. B 122, 67 (2005).

[18] R.K. Gupta, K. Ghosh, R. Patel, S.R Mishra, P.K. Kahol, Appl. Surf. Sci. 254, 5868 (2008).

[19] K. Manickathai, S. Kasi Viswanathan, M. Alagar, Indian J. Pure Appl. Phys. 46, 561 (2008).

[20] Zhiyong Zhao, D.L. Morel, C.S. Ferekides, Thin Solid Films 413, 203 (2002).

[21] R. Kumaravel, S. Menaka, S. Regina Mary Snega, K. Ramamurthi, K. Jeganathan, Mater. Chem. Phys. 122, 444 (2010).

[22] H. Zhu, D. Yang, G. Yu, H. Zhang, K. Yao, Nanotechnology 17, 2386 (2006).

[23] H.M. Deng, J.M. Hossenlopp, J. Phys. Chem. B 109 $66(2005)$.

[24] A.S. Maan, D.R. Goyal, K. Sachin Sharma, T.P. Sharma, J. Phys. III 4, 493 (1994).

[25] B. Saha, S. Das, K.K. Chattopadhyay, Sol. Energy. Mater. Sol. C 91, 1692 (2007).

[26] C.M. Liu, X.T. Zu, Q.M. Wei, L.M. Wang, J. Phys. D, Appl. Phys. 39, 2494 (2006).

[27] N. Clament Sagaya Selvam, R. Thinesh Kumar, K. Yogeenth, L. John Kennedy, G. Sekaran, J. Judith Vijaya, Powder. Technol. 211, 250 (2011).

[28] R. Ferro, J.A. Rodriguez, Thin Solid Films 347, 295 (1999).

[29] K. Gurumurugan, D. Mangalaraj, S.K. Narayandass, K. Sekar, C.P. Girija Vallabhan, Semicond. Sci. Technol. 9, 1827 (1994).

[30] R.R. Salunkhe, D.S. Dhawale, T.P. Gujar, C.D. Lokhande, Mater. Res. Bull. 44, 364 (2009).

[31] B. Malecka, A. Lacz, Thermochim. Acta 479, 12 (2008).
[32] C. Aydın, A. Omar, A.A. Al-Hartomy, F. AlGhamdi, I.S. Al-Hazmi, F. Yahia, F. El-Tantawy, J. Yakuphanoglu, J. Electroceram. 29, 155 (2012).

[33] S. Kose, F. Atay, V. Bilgin, I. Akyuz, Int. J. Hydrogen. Energ. 34, 5260 (2009).

[34] F. Yakuphanoglu, S. Ilican, M. Caglar, Y. Caglar, Superlatt. Microstruct. 47, 732 (2010).

[35] M.A. Gondala, Q.A. Drmosha, T.A. Salehb, Appl. Surf. Sci. 256, 7067 (2010).

[36] F. Gu, S.F. Wang, M.K. Lu, X.F. Cheng, S.W. Liu, G.J. Zhou, D. Xu, D.R. Yuan, J. Cryst. Growth 262, 182 (2004).

[37] M. Zaien, K. Omar, Z. Hassan, Int. J. Phys. Sci. 6 , 4176 (2011).

[38] M. Tadjarodi, A. Imani, Mater. Lett. 65, 1025 (2011).

[39] T. Krishnakumar, R. Jayaprakash, D. Sathiyaraj, N. Donnato, M. Latino, G. Neri, Sci. Adv. Mater. 2, 1 (2010). 